Notre Dame Journal of Formal Logic Volume 21, Number 4, October 1980

\title{
Number Theory for the Ordinals With a New Definition for Multiplication
}

\author{
HARRY GONSHOR
}

Introduction At the International Mathematics Congress in Helsinki (1978), Conway introduced a new operation on the ordinals which is slightly different from ordinary multiplication. This operation is obtained from natural addition in the same manner that multiplication is obtained from ordinary addition. The aim of this paper is to compare the nature of the primes and the factorization into primes in this system with that of the usual system.

Preliminaries Natural addition $\oplus$ is defimed as follows: If $\alpha=\omega^{a_{1}} b_{1}+$ $\omega^{a_{2}} b_{2}+\ldots+\omega^{a_{n}} b_{n}$ and $\beta=\omega^{a_{1}} c_{1}+\omega^{a_{2}} c_{2}+\ldots+\omega^{a_{n}} c_{n}$ then $\alpha \oplus \beta=\omega^{a_{1}}\left(b_{1}+\right.$ $\left.c_{1}\right)+\omega^{a_{2}}\left(b_{2}+c_{2}\right)+\ldots+\omega^{a_{n}}\left(b_{n}+c_{n}\right)$, i.e., instead of having any absorption, the operation resembles addition of ordinary polynomials.

We can now define by induction an operation $X$ as follows:

1. $\alpha \times(\beta+1)=(\alpha \times \beta) \oplus \alpha$

2. If $\beta$ is a limit ordinal $\alpha \times \beta=\operatorname{lub}_{\gamma<\beta}(\alpha \times \gamma)$.

To avoid any possible confusion the symbol $\cdot$ will be used to denote ordinary multiplication.

It is easy to see that $X$ is associative and distributive with respect to $\oplus$. Furthermore, the multiplication table takes on the following form:

1. $\left(\omega^{a_{1}} b_{1}+\omega^{a_{2}} b_{2}+\ldots+\omega^{a_{n}} b_{n}\right) \times c=\omega^{a_{1}} b_{1} c+\omega^{a_{2}} b_{2} c+\ldots+\omega^{a_{n}} b_{n} c$, if $c$ is finite.

2. $\left(\omega^{a_{1}} b_{1}+\omega^{a_{2}} b_{2}+\ldots+\omega^{a_{n}} b_{n}\right) \times \omega^{r}=\omega^{a_{1}+r}$.

Thus $\alpha \times \beta=\alpha \beta$ if $\beta$ is a limit ordinal, but if $\beta$ is a finite ordinal $c$, all the coefficients in the expansion of $\beta$ are multiplied by $c$, in contrast to ordinary multiplication where only the first coefficient is multiplied by $c$. 
We shall next see how this latter difference affects the classification of the primes.

Classification of the primes For ordinary multiplication it is well-known that the set of primes consists of the following three types:

1. The ordinary finite primes

2. $\delta$ numbers; i.e., ordinals of the form $\omega^{\omega^{a}}$

3. ordinals of the form $\omega^{a}+1$.

Trivially, the situation remains unchanged for types 1 and 2. The only case that need be considered is that of nonlimit ordinals larger than $\omega$.

\section{Lemma 1 All such primes are binomial.}

Proof: Suppose $\alpha=\omega^{a_{1}} b_{1}+\omega^{a_{2}} b_{2}+\ldots+b_{n}$. Then $\alpha=\left(\omega^{a_{n-1}} b_{n-1}+b_{n}\right)$. $\left(\omega^{a_{1}-a_{n-1}} b_{1}+\omega^{a_{2}-a_{n-1}} b_{2}+\ldots+1\right)$. Since the constant term is 1 , "." may be replaced by " $X$ ". Hence if $\alpha$ is not binomial, we have exhibited a factorization of $\alpha$.

Lemma 2 If $\omega^{a_{1}} b_{1}+b_{2}$ is prime then $b_{2}=1$.

Proof: $\omega^{a_{1}} b_{1}+b_{2}=b_{2} \cdot\left(\omega^{a_{1}} b_{1}+1\right)=b_{2} \times\left(\omega^{a_{1}} b_{1}+1\right)$.

Lemma $3 \quad \omega^{a} b+1$ is a prime.

Proof: The only possible way for a binomial nonlimit ordinal to factor is as finite ordinals times a binomial. Any finite factor must be a factor of the final term and hence must be 1 . Thus $\omega^{a} b+1$ is a prime.

Note. $\omega^{a} b+1=\left(\omega^{a}+1\right) \cdot b$. However $\left(\omega^{a}+1\right) \times b=\omega^{a} b+b$. Thus with respect to $\mathrm{X}, \omega^{a} b+1$ is a prime for all finite $b$ unlike the case for ordinary multiplication.

Factorization into primes First, we consider the existence question. Let $\alpha=\omega^{a_{1}} b_{1}+\omega^{a_{2}} b_{2}+\ldots+\omega^{a_{n}} b_{n}$. As for ordinary multiplication we can factor out $\omega^{a_{n}}$ and then factor $\omega^{a_{n}}$ into $\delta$ numbers. We are thus left with a nonlimit ordinal. By using the computation in Lemma 2 and induction in Lemma 1 we obtain a factorization into primes. Thus we obtain the following theorem:

Theorem 1 Every ordinal factors into primes with respect to $X$. Furthermore the factorization may be chosen so that every finite ordinal precedes every nonlimit infinite ordinal.

Note. The last remark is clear from the factorization in Lemma 1 since the second factor has final term 1 .

We are now ready to examine the uniqueness question. Since the contribution of $\delta$ numbers is essentially the same as it is for ordinary multiplication we restrict ourselves to nonlimit ordinals.

Theorem 2 Every nonlimit ordinal has a unique factorization in the form where all finite-ordinals precede all nonlimit ordinals. The order is unique except (of course) for the relative order of the constants. 
Proof: Consider $d \times\left(\omega^{r_{1}} a_{1}+1\right) \times\left(\omega^{r_{2}} a_{2}+1\right) \times \ldots \times\left(\omega^{r_{n}} a_{n}+1\right)$. This has the form $\omega^{r_{1}+r_{2}+\ldots+r_{n}} a_{n}+\omega^{r_{1}+r_{2}+\ldots+r_{n-1}} a_{n-1}+\ldots+\omega^{r_{1}} a_{1}+d$. By the uniqueness of representation of ordinals it is clear that the product determines the factors uniquely.

Finally it is clear from the existence theorem that uniqueness fails in general. In fact to be specific, uniqueness will fail for any nonlimit ordinal which can be expressed as a product in which there are finite ordinals following nonlimit ordinals. It is easy to see that a nonlimit ordinal $\alpha=\omega^{a_{1}} b_{1}+\omega^{a_{2}} b_{2}+$ $\ldots+\omega^{a_{n}} b_{n}+d$ has that property iff $\operatorname{gcd}\left(b_{n}, d\right)>1$. In fact any finite ordinal that follows a nonlimit ordinal in a factorization of $\alpha$ necessarily divides both $b_{n}$ and $d$ as is clear from the multiplication table. Conversely a common factor $c$ of $b_{n}$ and $d$ gives rise to a factorization of $\omega^{a_{n}} b_{n}+d$ with $c$ on the right. This in turn leads to a factorization of $\alpha$. Because of absorption on one side and not on the other when multiplying by a finite ordinal, the factorizations are distinct (not only in order).

As an example $\omega \cdot 2+2=(\omega+1) \times 2=2 \times(\omega \cdot 2+1)$. Of course for ordinary multiplication $\omega \cdot 2+2=2 \cdot(\omega+1) \cdot 2$. Thus we have the following theorem:

Theorem $3 \quad$ A nonlimit ordinal $\alpha=\omega^{a_{1}} b_{1}+\omega^{a_{2}} b_{2}+\ldots+\omega^{a_{n}} b_{n}+d$ factors uniquely into primes with respect to $X$ iff $\operatorname{gcd}\left(b_{n}, d\right)=1$.

Note: More factorizations are possible if $\operatorname{gcd}\left(b_{n-1}, b_{n}, d\right)>1$. In fact, more generally, it is clear and left to the reader to see how a common factor of $d, b_{n}, b_{n-1}, \ldots, b_{n-r}$ leads to a factorization.

\section{Department of Mathematics}

Rutgers College

New Brunswick, New Jersey 08903 